\title{
Selecting for BRCAI testing using a combination of homogeneous selection criteria and immunohistochemical characteristics of breast cancers
}

\author{
GianMaria Miolo*1,2, Vincenzo Canzonieri³ ${ }^{\text {, Clelia De Giacomi², }}$ \\ Lara Della Puppa ${ }^{1}$, Riccardo Dolcetti ${ }^{4}$, Davide Lombardi ${ }^{2}$, Tiziana Perin ${ }^{3}$, \\ Simona Scalone ${ }^{2}$, Andrea Veronesi ${ }^{2}$ and Alessandra Viel ${ }^{1}$
}

\begin{abstract}
Address: ${ }^{1}$ Department of Molecular Oncology and Translational Research, Division of Experimental Oncology 1, Centro di Riferimento Oncologico, National Cancer Institute, Aviano, Italy, ${ }^{2}$ Department of Senology, Division of Medical Oncology C, Centro di Riferimento Oncologico, National Cancer Institute, Aviano, Italy, ${ }^{3}$ Department of Diagnostic Laboratories and Cell Therapy, Division of Pathology, Centro di Riferimento Oncologico, National Cancer Institute, Aviano, Italy and ${ }^{4}$ Department of Medical Oncology, Cancer Biommunotherapy Unit, Centro di Riferimento Oncologico, National Cancer Institute, Aviano, Italy

Email: GianMaria Miolo* - gmiolo@libero.it; Vincenzo Canzonieri - vcanzonieri@cro.it; Clelia De Giacomi - cdegiacomi@cro.it; Lara Della Puppa - ldellapuppa@cro.it; Riccardo Dolcetti - rdolcetti@cro.it; Davide Lombardi - dlombardi@cro.it; Tiziana Perin - tperin@cro.it; Simona Scalone - sscalone@cro.it; Andrea Veronesi - averonesi@cro.it; Alessandra Viel - aviel@cro.it

* Corresponding author
\end{abstract}

Published: 10 October 2009

BMC Cancer 2009, 9:360 doi:10.1 I86/I47I-2407-9-360

This article is available from: http://www.biomedcentral.com/147I-2407/9/360

(c) 2009 Miolo et al; licensee BioMed Central Ltd.

This is an Open Access article distributed under the terms of the Creative Commons Attribution License (http://creativecommons.org/licenses/by/2.0), which permits unrestricted use, distribution, and reproduction in any medium, provided the original work is properly cited.

\begin{abstract}
Background: BRCAI gene-related tumours are more frequently estrogen receptor (ER) and progesterone receptor (PR) negative with a lower prevalence of human epidermal growth factor receptor 2 (HER2) overexpression or amplification. We evaluated the effectiveness of a combination of homogeneously selected criteria and immunohistochemical $(\mathrm{IHC})$ characteristics of Familial Breast Cancers (FBCs) in detecting BRCAI mutation carriers.
\end{abstract}

Methods: Primary breast tumours from 93 FBC patients defined by specific eligibility criteria, based on personal and familial tumour history, were evaluated by Allred's method. The BRCAI molecular analysis, including Multiplex Ligation-dependent Probe Amplification (MLPA), was considered as the gold standard assay.

Results: A total of IO BRCAI pathogenetic mutations was found. With the exclusion of the tumours characterized by double positive receptorial status and/or strong HER2 positivity ( $3+$ ), we identified 22 patients, 10 of whom resulted as BRCAI mutation carriers. The sensitivity, specificity, positive and negative predictive values were $100 \%, 83.3 \%, 45.4 \%$ and $100 \%$ respectively.

Conclusion: Our findings suggest that the IHC analysis by Allred's method improves our ability to select patients for BRCAI testing. 


\section{Background}

One of the still unexplained features of BRCA1-mutant breast tumours is their frequent lack of ER expression; in fact, up to $90 \%$ of these tumours exhibit a loss of ER expression [1,2]. In addition, BRCA1-associated breast cancers frequently show a low prevalence of HER2 overexpression or amplification [3-5].

Patients with a strong family history of breast and/or ovarian cancer are counselled to receive genetic testing for mutations in the BRCA1/BRCA2 genes. The selection of patients is crucial and relies mostly on family history and phenotype ascertainment [6-8]. In general, only $20-40 \%$ of screened families, even using rigid selection criteria, have positive mutation results $[9,10]$. In particular, the rate of mutations in Italian breast and/or ovarian cancer families is rather controversial and ranges from $8 \%$ to $37 \%$ [11]. Moreover, it has been recently pointed out that only less than $10 \%$ of cases with a positive family history harbour a BRCA1 mutation [12].

In this study, we evaluated the effectiveness of a combination of selected eligibility criteria and IHC characteristics of breast cancer to identify patients who have a higher probability of being BRCA1 mutation carriers.

\section{Methods}

From September 2002 to October 2008, a total of 93 female index cases affected with breast cancer complying with the eligibility criteria for BRCA testing in use at the Centro di Riferimento Oncologico, National Cancer Institute, Aviano, Italy were enrolled in the study [13]. Cases affected with ovarian cancer and male breast cancer were excluded. Written informed consent for genetic testing was obtained from all the participants. The study fully conformed to the Declaration of Helsinki and to the current Italian Legislation and was also approved by the Ethical Committee of the Institute.

Genomic DNA was purified from blood samples obtained from each proband. Screening for mutations in the BRCA1/BRCA2 genes was carried out by Denaturing High Performance Liquid Cromatography and direct sequencing techniques. Primer sequences and PCR conditions have been previously described [14-16]. Germline rearrangements of BRCA1/BRCA2 genes were also analysed by Multiple Ligation-dependent Probe Amplification (MLPA). Missense, silent, and intronic mutations, whose clinical significance have not yet been reported, were classified as genetic variants of uncertain significance (unclassified variant, uv) in accordance with the BIC database [17].

Ventana Medical Systems clone 6F11 (Ventana Medical Systems, Inc., Tucson, AZ) for the evaluation of the ER and
Ventana Medical Systems clone 1E2 for the evaluation of the PR were used. With the Allred score, the proportion score (PS) and the intensity score (IS) are assessed in six and four grades, respectively $0-5$ and $0-3$, then the total score is assessed in eight grades ( 0 and 2-8) [18]. Tumours with an IHC total score of 3 or more were reported as positive. The IHC analysis of HER2 was performed by staining with the rabbit anti-human HER2 antibody clone 4B5 (Ventana Medical Systems). A score index of 0, 1, 2 and 3 was used, corresponding respectively to negative, weak, moderate and strong staining intensity, and the percentage of positive cells at each intensity was estimated subjectively.

The sensitivity, specificity, and positive and negative predictive values were calculated from the results of the IHC analysis to predict for BRCA1 mutations.

\section{Results}

In total, 19 different BRCA1/BRCA2 deleterious mutations were identified in the 93 unrelated patients. Ten (10.8\%) patients resulted BRCA1 mutation carriers whereas in nine $(9.7 \%)$ a BRCA2 mutation was found (Tables 1 and 2). The prevalence of BRCA1 large genomic rearrangements accounted for $20 \%$ (2/10) of the BRCA1 gene mutation spectrum. No large deletions or duplications involving the BRCA2 gene were observed. In this series, all BRCA1 tumours showed a double or a single negative hormonal receptorial status, whereas HER2 was invariably negative or only weakly expressed (Table 1).

Altogether, 21 cases showed an uv alteration of BRCA1 and/or BRCA2 genes: 7 BRCA1 uv, 7 BRCA2 uv, 5 cases with a variant in both genes, 1 triple BRCA1 uv and 1 double BRCA2 uv associated with a BRCA1 uv, respectively (Table 3).

To evaluate the sensitivity and specificity of the IHC screening in a selected set of patients, the combination between BRCA1 point mutation screening and MLPA was considered as the gold standard assay. According to the receptorial status, patients were divided into two groups. The first group included all the ER negative and/or PR negative tumours, whereas the second group consisted of the double positive ones. The BRCA1/2 negative mutation group is shown in Table 4.

Twenty-nine tumours did not show a positive staining for ER and/or PR hormone receptors. Notably, 10 of these negative cases were from BRCA1 mutation carriers (Table 5). Furthermore, not considering the cases showing a strong HER2 positivity (3+), the mutation screening could have been performed in only 22 patients, detecting the same number of BRCA1 mutations (Table 6). 
Table I: BRCAI group and IHC tumour characteristics.

\begin{tabular}{|c|c|c|c|c|c|c|c|c|c|c|c|c|}
\hline ID pts & $\begin{array}{c}\text { Age at } \\
\text { diagnosis }\end{array}$ & DNA & Mutation effect & Grade & ${ }^{\circ} \mathrm{ER}$ (score) & ${ }^{\circ}$ PR (score) & §HER2 & $\begin{array}{c}\text { ^ BRCAPRO } \\
\text { BRCAI }\end{array}$ & $\begin{array}{c}\hat{\wedge} \\
\text { BRCAP } \\
\text { RO } \\
\text { BRCA2 }\end{array}$ & $\begin{array}{c}\hat{\wedge} \\
\text { BRCAP } \\
\text { RO } \\
\text { total }\end{array}$ & $\begin{array}{c}* \mathrm{BC} \text { and } \mathrm{CBC} \\
\text { cases }\end{array}$ & $\begin{array}{l}* \text { OC } \\
\text { cases }\end{array}$ \\
\hline BR332 & 42 & 633delC & $233 x$ & 3 & $-(2)$ & $-(0)$ & - & 0.975 & 0.013 & 0.987 & I (27) & $\begin{array}{c}2 \\
(35,56)\end{array}$ \\
\hline BR334 & 26 & $2428 C>A$ & S770X & 3 & $-(0)$ & - (0) & - & 0.658 & 0.091 & 0.748 & I (53) & I (75) \\
\hline BR34I & 45 & del 9-19 & del ex. $9-19$ ter 184 & 3 & $-(0)$ & $-(0)$ & - & 0.320 & 0.524 & $0.84 I$ & $\left.\begin{array}{c}3 \\
(37,48 \\
M\end{array}, 52\right)$ & I \\
\hline BR352 & 37 & del 23-24 & unpredictable & 3 & $-(0)$ & $-(0)$ & $1+$ & 0.777 & 0.075 & 0.850 & $\begin{array}{c}\mathrm{I} \\
(29+42 \mathrm{CBC})\end{array}$ & I \\
\hline BR384 & $50+56 \mathrm{CBC}$ & 795delT & $233 x$ & 3 & $-(0)$ & $-(0)$ & $\mathrm{I}+/ 2+$ & 0.529 & 0.085 & 0.613 & $2(56,57)$ & I (57) \\
\hline BR386 & 40 & $1806 \mathrm{C}>\mathrm{T}$ & Q563X & 3 & $-(0)$ & - (0) & $1+/ 2+$ & 0.311 & 0.048 & 0.358 & 1 & I (69) \\
\hline BR392 & 31 & 795delT & $233 x$ & 3 & $-(0)$ & $-(0)$ & - & 0.813 & 0.066 & 0.876 & $\begin{array}{c}\mathrm{I} \\
(46+48 \mathrm{CBC})\end{array}$ & 1 \\
\hline BR44I & 46 & $5|8|$ delGTT & I688delV & 3 & $-(0)$ & $-(0)$ & - & 0.166 & 0.036 & 0.202 & $3(46,47,48)$ & I (63) \\
\hline BR507 & 22 & 633delC & $233 x$ & 3 & - $(0)$ & $-(0)$ & $1+$ & 0.091 & 0.015 & 0.106 & 1 & 1 \\
\hline BR587 & $38+60 \mathrm{CBC}$ & $300 T>G$ & C6IG & 3 & $-(0)$ & $-(0)$ & $1+$ & 0.922 & 0.063 & 0.984 & $2(40,43)$ & I (60) \\
\hline
\end{tabular}

$\mathrm{BC}=$ breast cancer; $\mathrm{CBC}=$ controlateral breast cancer; $\mathrm{OC}=$ ovarian cancer; $/=$ no cases; $M=$ male. The ages at diagnosis of the first cousins are in boldface type; ${ }^{\circ} \mathrm{IHC}$ Allred total scores of 3 or more were reported as positive. §HER2 IHC scores: - negative, I+ weak, 2+ moderate, $3+$ strong. ^ Probability of mutation calculated by BRCAPRO software http://www.isds.duke.edu/ gp/ brcapro.html; * number of additional tumours in the family and ages of onset in parentheses. 


\begin{tabular}{|c|c|c|c|c|c|c|c|c|c|c|c|c|}
\hline ID pts & $\begin{array}{c}\text { Age at } \\
\text { diagnosis }\end{array}$ & DNA & $\begin{array}{c}\text { Mutation } \\
\text { effect }\end{array}$ & Grade & ${ }^{\circ}$ ER (score) & ${ }^{\circ} \mathrm{PR}$ (score) & §HER2 & $\begin{array}{c}\text { A BRCAPRO } \\
\text { BRCAI }\end{array}$ & $\begin{array}{c}\text { A BRCAPRO } \\
\text { BRCA2 }\end{array}$ & $\begin{array}{c}\text { A BRCAPRO } \\
\text { total }\end{array}$ & $\begin{array}{c}\text { *BC, CBC and } \\
\text { BBC cases }\end{array}$ & $\begin{array}{l}* \text { OC } \\
\text { cases }\end{array}$ \\
\hline BR288 & 36 & 9326insA & $3043 x$ & 3 & $+(6)$ & $+(5)$ & $1+$ & 0.003 & 0.026 & 0.029 & I & I (44) \\
\hline BR295 & 54 & 657ins C & $157 X$ & 2 & $+(8)$ & $+(5)$ & - & 0.029 & 0.405 & 0.434 & $\stackrel{2}{2}(4 I+43$ CBC, 48$)$ & I \\
\hline BR312 & 47 & IVSI6-2A>G & Exon skipping & 3 & $+(5)$ & $+(3)$ & $1+$ & 0.002 & 0.365 & 0.367 & $\begin{array}{c}2 \\
(47,77 \mathrm{M})\end{array}$ & I \\
\hline BR342 & $44+52 \mathrm{CBC}$ & $9106 \mathrm{C}>\mathrm{T}$ & Q2960X & 3 & $+(8)$ & $+(3)$ & $2+$ & 0.006 & 0.182 & 0.187 & $2(59,68+75 \mathrm{CBC})$ & I \\
\hline BR360 & 43 & IVS23+IG >T & Exon skipping & 3 & $+(4)$ & $+(6)$ & $2+/ 3+$ & 0.088 & 0.553 & 0.640 & $\begin{array}{c}\mathrm{I} \\
(30 \mathrm{BBC})\end{array}$ & I \\
\hline BR382 & $44+63 \mathrm{CBC}$ & 7507del7 & $2168 X$ & 3 & $+(6)$ & $+(8)$ & $1+$ & 0.002 & 0.061 & 0.063 & I (35) & I \\
\hline BR4IO & 24 & $9325 \mathrm{del} A$ & $3061 X$ & 2 & $+(7)$ & $+(4)$ & $1+$ & 0.170 & 0.557 & 0.727 & I (47) & $\begin{array}{c}2 \\
(54,73)\end{array}$ \\
\hline BR450 & 48 & $4450 \mathrm{C}>\mathrm{T}$ & QI408X & 1 & $+(7)$ & $+(7)$ & - & 0.010 & 0.044 & 0.054 & $2(52,55)$ & $\begin{array}{c}2 \\
(38,55)\end{array}$ \\
\hline BR57I & 31 & 3036del4 & $959 x$ & 2 & $+(3)$ & $+(6)$ & $3+$ & 0.001 & 0.037 & 0.038 & $\begin{array}{c}3 \\
(50,50,61)\end{array}$ & I \\
\hline
\end{tabular}

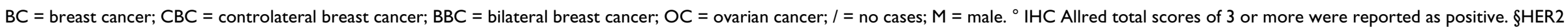

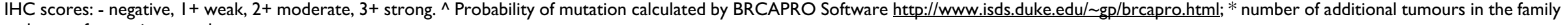
and ages of onset in parentheses. 
Table 3: BRCAI and/or BRCA2 uv group and IHC tumour characteristics.

\begin{tabular}{|c|c|c|c|c|c|c|c|c|c|c|c|c|c|}
\hline & ID pts & $\begin{array}{c}\text { Age at } \\
\text { diagnosis }\end{array}$ & DNA & $\begin{array}{c}\text { Mutation } \\
\text { effect }\end{array}$ & Grade & $\begin{array}{c}{ }^{\circ} \text { ER } \\
\text { (score) }\end{array}$ & $\begin{array}{c}{ }^{\circ} \mathrm{PR} \\
\text { (score) }\end{array}$ & §HER2 & $\begin{array}{c}\hat{A} \\
\text { BRCAPRO } \\
\text { BRCAI }\end{array}$ & $\begin{array}{c}\hat{\wedge} \\
\text { BRCAPRO } \\
\text { BRCA2 }\end{array}$ & $\begin{array}{c}\hat{\hat{A}} \\
\text { BRCAPR } \\
\text { O total }\end{array}$ & $\begin{array}{c}\mathrm{BC}, \mathrm{CBC} \\
\text { and } \mathrm{BBC} \\
\text { cases }\end{array}$ & OC cases \\
\hline \multicolumn{14}{|l|}{ BRCAI uv } \\
\hline & BR4I7 & 30 & $1186 A>G$ & Q356R & 2 & $+(3)$ & $-(0)$ & $3+$ & 0.015 & 0.040 & 0.055 & I (46) & 1 \\
\hline & BR427 & 48 & $4274 A>G$ & LI385L & 2 & $+(7)$ & $+(7)$ & $1+$ & 0.000 & 0.010 & 0.011 & I (44) & 1 \\
\hline & BR437 & 50 & $235 I T>G$ & A744A & 1 & $+(5)$ & $+(3)$ & $1+$ & 0.000 & 0.014 & 0.015 & $\begin{array}{c}3(50,55 \\
59)\end{array}$ & 1 \\
\hline & BR523 & $48 \mathrm{BBC}$ & $\begin{array}{c}\text { IVS20+60ins } \\
12\end{array}$ & & 2 & $+(6)$ & $+(7)$ & $1+$ & 0.018 & 0.245 & 0.263 & $3(50,50,52)$ & 1 \\
\hline & BR570 & 47 & $522 I T>C$ & LI70IP & 3 & $-(0)$ & $+(3)$ & $3+$ & 0.094 & 0.169 & 0.264 & $\begin{array}{l}2(49+52 \\
C B C, 56)\end{array}$ & 1 \\
\hline & BR647 & 49 & $1186 A>G$ & Q356R & 3 & $+(8)$ & $+(7)$ & $1+$ & 0.005 & 0.010 & 0.015 & $\begin{array}{c}3(65,67 \\
70)\end{array}$ & 1 \\
\hline & BR658 & 43 & $1186 A>G$ & Q356R & 3 & $+(6)$ & $+(6)$ & $2+$ & 0.001 & 0.069 & 0.070 & $\begin{array}{c}3(50+63 \\
C B C, 57 \\
60 *)\end{array}$ & 1 \\
\hline & \multirow[t]{3}{*}{ BR665 } & $65 \mathrm{BBC}$ & $655 A>G$ & YI79C & 2 & $+(5)$ & $+(5)$ & - & 0.001 & 0.004 & 0.004 & $\begin{array}{c}3 \\
(60,80,80)\end{array}$ & 1 \\
\hline & & & I575T $>C$ & $\mathrm{~F} 486 \mathrm{~L}$ & & & & & & & & & \\
\hline & & & $1767 A>G$ & $\mathrm{~N} 550 \mathrm{H}$ & & & & & & & & & \\
\hline \multicolumn{14}{|l|}{ BRCA2 uv } \\
\hline & BR376 & 55 & IVS2-7T>A & & 2 & $+(6)$ & $+(3)$ & $1+$ & 0.000 & 0.008 & 0.008 & $\begin{array}{c}2 \\
(45,90)\end{array}$ & 1 \\
\hline & BR390 & $\begin{array}{c}51 \mathrm{OC}+54 \\
\mathrm{BBC}\end{array}$ & $6328 C>T$ & R2034C & 2 & $+(5)$ & $+(8)$ & - & 0.130 & 0.648 & 0.777 & $\begin{array}{c}3(34,39 \\
40)\end{array}$ & 1 \\
\hline & BR458 & $44+49 \mathrm{CBC}$ & $5972 T>C$ & T1952I & 2 & $+(7)$ & $+(5)$ & - & 0.025 & 0.433 & 0.459 & $\begin{array}{c}2(50 \mathrm{BBC}, \\
70)\end{array}$ & 1 \\
\hline & BR486 & 56 & $\begin{array}{l}2457 \mathrm{~T}>\mathrm{Cl} \\
9329 \mathrm{~A}>\mathrm{G}\end{array}$ & $\begin{array}{l}\mathrm{H} 743 \mathrm{H} / \\
\mathrm{R} 3034 \mathrm{Q}\end{array}$ & 3 & $+(5)$ & $+(8)$ & - & 0.000 & 0.000 & 0.000 & $\begin{array}{c}4(\mathbf{5 7}, \mathbf{7 1}, \\
75,90)\end{array}$ & 1 \\
\hline
\end{tabular}


Table 3: BRCAI and/or BRCA2 uv group and IHC tumour characteristics. (Continued)

\begin{tabular}{|c|c|c|c|c|c|c|c|c|c|c|c|c|}
\hline BR505 & 67 & $675 \mid C>G$ & Q2175E & 1 & $+(7)$ & $+(7)$ & - & 0.018 & 0.426 & 0.444 & $\begin{array}{c}4(40 \mathrm{BBC}, \\
50 \mathrm{BBC}, 53 \\
\mathrm{BBC}, 58)\end{array}$ & 1 \\
\hline BR538 & 62 & $4236 \mathrm{G}>\mathrm{A}$ & LI356L & 2 & $+(7)$ & $+(7)$ & - & 0.001 & 0.025 & 0.026 & $\begin{array}{c}2(50 \mathrm{BBC}, \\
56)\end{array}$ & 1 \\
\hline BR587 & $38+60 \mathrm{CBC}$ & $\begin{array}{l}\text { IVS24- } \\
\text { II } 3 T>G\end{array}$ & & 3 & $-(0)$ & $-(0)$ & $1+$ & 0.922 & 0.063 & 0.984 & $2(40,43)$ & I (60) \\
\hline \multicolumn{13}{|l|}{$\begin{array}{l}\text { BRCAI/2 } \\
\text { uv }\end{array}$} \\
\hline BR5।4 & 57 & $3238 G>A$ & SI040N & I & $+(7)$ & $+(8)$ & - & 0.001 & 0.061 & 0.062 & $\begin{array}{c}4(42,45 \\
49,55)\end{array}$ & 1 \\
\hline & & $\begin{array}{c}\text { IVS4+67A> } \\
C \\
|6| 3 A>G\end{array}$ & E462G & & & & & & & & & \\
\hline BR552 & 70 & $4158 A>G$ & RI347G & 3 & $+(5)$ & $+(3)$ & $3+$ & 0.000 & 0.012 & 0.012 & $\begin{array}{c}3 \\
(40,47,65)\end{array}$ & 1 \\
\hline & & $6328 \mathrm{C}>\mathrm{T}$ & R2034C & & & & & & & & & \\
\hline BR567 & 50 & $\begin{array}{c}\text { IVS4+67A> } \\
C\end{array}$ & & 3 & $+(6)$ & $+(7)$ & - & 0.009 & 0.215 & 0.225 & $\begin{array}{c}3(37 \mathrm{BBC}, \\
50 \mathrm{BBC}, 50)\end{array}$ & I (59) \\
\hline & & $2457 T>C$ & $\mathrm{H} 743 \mathrm{H}$ & & & & & & & & & \\
\hline BR57I & 31 & IVS9-34T>C & & 2 & $+(3)$ & $+(6)$ & $3+$ & 0.001 & 0.037 & 0.038 & $\begin{array}{c}3(50,50 \\
61)\end{array}$ & I \\
\hline & & $5972 \mathrm{C}>\mathrm{T}$ & TI95IM & & & & & & & & & \\
\hline BR580 & 43 & $3238 \mathrm{G}>\mathrm{A}$ & SI040N & 3 & $+(7)$ & $+(5)$ & $1+$ & 0.001 & 0.030 & 0.031 & $\begin{array}{c}2 \\
(46,68)\end{array}$ & I \\
\hline & & $1409 A>G$ & E394A & & & & & & & & & \\
\hline BR640 & 30 & $855 T>G$ & L246V & 3 & $+(7)$ & $-(0)$ & $1+$ & 0.117 & 0.261 & 0.378 & $\begin{array}{c}2 \\
(30,40)\end{array}$ & I \\
\hline & & $4296 \mathrm{G}>\mathrm{A}$ & LI356L & & & & & & & & & \\
\hline
\end{tabular}

$\mathrm{BC}=$ breast cancer; $\mathrm{CBC}=$ controlateral breast cancer; $\mathrm{BBC}=$ bilateral breast cancer; $\mathrm{OC}=$ ovarian cancer; $/=$ no cases; $\mathrm{M}=$ male. The ages at diagnosis of the first cousins are in boldface type - IHC Allred total scores of 3 or more were reported as positive. \$HER2 IHC scores: - negative, I+ weak, 2+ moderate, 3+ strong. ^ Probability of mutation calculated by BRCAPRO software http://www.isds.duke.edu/ gp/brcapro.html; * number of additional tumours in the family and ages of onset in parentheses. 
Table 4: non BRCAI/2 mutation group and IHC tumour characteristics.

\begin{tabular}{|c|c|c|c|c|c|c|c|c|c|c|}
\hline ID pts & $\begin{array}{c}\text { Age at } \\
\text { diagnosis }\end{array}$ & Grade & ${ }^{\circ}$ ER (score) & ${ }^{\circ} \mathrm{PR}$ (score) & §HER2 & $\begin{array}{l}\text { ^ BRCAPRO } \\
\text { BRCAI }\end{array}$ & $\begin{array}{l}\text { ^ BRCAPRO } \\
\text { BRCA2 }\end{array}$ & $\begin{array}{c}\text { A BRCAPRO } \\
\text { total }\end{array}$ & $\begin{array}{l}\text { *BC, CBC and } \\
\text { BBC cases }\end{array}$ & *OC cases \\
\hline BR27I & 40 & G2 & $+(7)$ & $+(5)$ & $1+/ 2+$ & 0.002 & 0.036 & 0.038 & I (37) & 1 \\
\hline BR275 & $28 \mathrm{BBC}$ & G3 & $+(7)$ & $+(7)$ & - & 0.067 & 0.578 & 0.645 & I (55) & 1 \\
\hline BR28I & 42 & G3 & $+(6)$ & $+(4)$ & $3+$ & 0.008 & 0.222 & 0.230 & $\begin{array}{c}6(27,36,50,60,50 \\
\text { BBC, 60) }\end{array}$ & 1 \\
\hline BR297 & 54 & G3 & $-(0)$ & $-(0)$ & - & 0.064 & 0.018 & 0.081 & $2(42,69)$ & 1 \\
\hline BR304 & 41 & G3 & $+(7)$ & $+(6)$ & - & 0.002 & 0.058 & 0.060 & $2(52,64)$ & 1 \\
\hline BR315 & $46 \mathrm{BBC}$ & G2 & $+(7)$ & $+(7)$ & $1+$ & 0.012 & 0.160 & 0.172 & I (49) & 1 \\
\hline BR335 & 64 & G2 & $+(8)$ & $-(0)$ & $1+$ & 0.002 & 0.017 & 0.020 & $2(57,70)$ & I (69) \\
\hline BR338 & 36 & G3 & $-(0)$ & $-(0)$ & $3+$ & 0.131 & 0.029 & 0.160 & $2(45,71)$ & 1 \\
\hline BR339 & 74 & G2 & $+(7)$ & $+(7)$ & - & 0.003 & 0.085 & 0.088 & $\begin{array}{c}3 \\
(39,61+63 \mathrm{CBC} \\
66)\end{array}$ & $2(54,64)$ \\
\hline BR340 & 35 & G3 & $+(7)$ & $-(0)$ & $3+$ & 0.009 & 0.026 & 0.035 & $2(43,59)$ & 1 \\
\hline BR348 & 55 & G3 & $+(7)$ & $-(0)$ & $1+$ & 0.009 & 0.062 & 0.071 & $2(56,56)$ & I (68) \\
\hline BR350 & 42 & G3 & $+(7)$ & $+(7)$ & $2+$ & 0.001 & 0.014 & 0.015 & $\begin{array}{c}4 \\
(49,57,61 *, 70 *)\end{array}$ & 1 \\
\hline BR357 & 35 & G3 & $+(6)$ & $-(0)$ & - & 0.002 & 0.014 & 0.016 & $2(55,67)$ & 1 \\
\hline BR377 & 46 & G3 & $+(7)$ & $+(6)$ & - & 0.000 & 0.007 & 0.007 & $2(46,70)$ & 1 \\
\hline BR380 & 30 & G2 & $-(0)$ & $-(0)$ & $1+/ 2+$ & 0.303 & 0.067 & 0.370 & $3(42,64,64)$ & I \\
\hline BR38I & 45 & G3 & $+(5)$ & $+(6)$ & - & 0.000 & 0.004 & 0.004 & $2(45,85)$ & 1 \\
\hline BR398 & 50 & G2 & $+(5)$ & $+(3)$ & - & 0.000 & 0.020 & 0.020 & $\begin{array}{c}5 \\
(60,61,65,70,98)\end{array}$ & 1 \\
\hline
\end{tabular}




\begin{tabular}{|c|c|c|c|c|c|c|c|c|c|c|}
\hline BR403 & $53+57 \mathrm{CBC}$ & G3 & $+(7)$ & $+(7)$ & - & 0.000 & 0.006 & 0.007 & $\begin{array}{c}\mathrm{I} \\
(82+84 \mathrm{CBC})\end{array}$ & 1 \\
\hline BR408 & 53 & $\mathrm{G} 2$ & $+(6)$ & $+(4)$ & - & 0.000 & 0.002 & 0.002 & $\begin{array}{c}3 \\
\left(71^{*}, 85^{*}, 67^{\circ}\right)\end{array}$ & I \\
\hline BR4I4 & 60 & $\mathrm{G} 2$ & $-(2)$ & $+(4)$ & - & 0.003 & 0.101 & 0.104 & $2(73 M, 50)$ & I \\
\hline BR4I8 & 27 & G3 & $-(0)$ & $-(0)$ & $3+$ & 0.093 & 0.015 & 0.108 & l & l \\
\hline BR422 & 55 & G3 & $+(6)$ & $-(2)$ & - & 0.002 & 0.014 & 0.016 & $2(50,53)$ & I \\
\hline BR424 & 45 & $\mathrm{G} 2$ & $+(5)$ & $+(7)$ & $2+$ & 0.006 & 0.113 & 0.119 & $\begin{array}{c}\mathrm{I} \\
(49+59 \mathrm{CBC})\end{array}$ & 1 \\
\hline BR425 & 27 & $\mathrm{G} 2$ & $+(3)$ & $+(5)$ & $3+$ & 0.001 & 0.012 & 0.013 & l & l \\
\hline BR439 & 52 & G3 & $-(0)$ & $-(2)$ & - & 0.052 & 0.030 & 0.082 & $3(36,60,70)$ & I (78) \\
\hline BR445 & 54 & GI & $+(6)$ & $+(3)$ & $1+$ & 0.004 & 0.067 & 0.072 & $2(45,48+52 C B C)$ & l \\
\hline BR446 & 50 & $\mathrm{G} 2$ & $+(7)$ & $+(4)$ & $1+$ & 0.000 & 0.008 & 0.008 & I (40) & 1 \\
\hline BR447 & 65 & $\mathrm{G} 2$ & $+(8)$ & $+(6)$ & - & 0.001 & 0.045 & 0.046 & $2(58,79)$ & 1 \\
\hline BR449 & 50 & $\mathrm{G} 2$ & $+(6)$ & $+(7)$ & - & 0.001 & 0.020 & 0.021 & I (37) & 1 \\
\hline BR457 & 53 & G3 & $+(4)$ & $-(2)$ & $3+$ & 0.000 & 0.003 & 0.004 & $2(55,55)$ & I \\
\hline BR463 & 48 & G3 & $+(6)$ & $+(5)$ & - & 0.001 & 0.032 & 0.033 & I (67 BBC) & I \\
\hline BR480 & 53 & $\mathrm{G} 2$ & $+(7)$ & $+(7)$ & - & 0.004 & 0.135 & 0.139 & $3(31,50,53)$ & 1 \\
\hline BR484 & 57 & $\mathrm{G} 2$ & $+(7)$ & $+(7)$ & $1+$ & 0.000 & 0.014 & 0.014 & $\begin{array}{c}2 \\
(55,73+78 \text { CBC })\end{array}$ & l \\
\hline BR494 & 58 & G3 & $+(5)$ & $-(0)$ & - & 0.020 & 0.352 & 0.372 & $\begin{array}{c}4 \\
(40,43,45, \text { NA M })\end{array}$ & 1 \\
\hline BR498 & 46 & $\mathrm{G} 2$ & $+(5)$ & $+(7)$ & - & 0.005 & 0.837 & 0.841 & $\begin{array}{c}4 \\
(42 \mathrm{BBC}, 48,49 \mathrm{M}, \\
74)\end{array}$ & 1 \\
\hline BR506 & 55 & GI & - (0) & $+(5)$ & - & 0.000 & 0.012 & 0.012 & $3(62,30,76)$ & l \\
\hline
\end{tabular}


Table 4: non BRCAI/2 mutation group and IHC tumour characteristics. (Continued)

\begin{tabular}{|c|c|c|c|c|c|c|c|c|c|c|}
\hline BR535 & 56 & G3 & $+(7)$ & $+(7)$ & $1+$ & 0.000 & 0.019 & 0.019 & $3(47,53, N A)$ & 1 \\
\hline BR542 & 45 & G3 & $+(7)$ & $+(7)$ & $1+$ & 0.007 & 0.112 & 0.119 & $2(36,62)$ & I \\
\hline BR545 & 63 & G2 & $+(7)$ & $+(6)$ & $1+$ & 0.000 & 0.003 & 0.003 & $2(64,70)$ & 1 \\
\hline BR549 & 36 & G3 & $+(6)$ & $+(5)$ & $1+$ & 0.013 & 0.142 & 0.155 & I (36) & I \\
\hline BR553 & 54 & G2 & $+(7)$ & $+(6)$ & $1+$ & 0.011 & 0.486 & 0.497 & $2(49,65)$ & I (55) \\
\hline BR558 & 45 & GI & $+(7)$ & $+(8)$ & $1+$ & 0.000 & 0.029 & 0.030 & $3(48,78,78)$ & 1 \\
\hline BR560 & 78 & G2 & $+(7)$ & $+(3)$ & $1+$ & 0.000 & 0.025 & 0.025 & $3(45,48,78)$ & 1 \\
\hline BR569 & 41 & G2 & $+(7)$ & $+(8)$ & - & 0.114 & 0.072 & 0.185 & 1 & $2(60,60)$ \\
\hline BR582 & 27 & G3 & $+(6)$ & $+(8)$ & - & 0.001 & 0.016 & 0.016 & I & I \\
\hline BR584 & 42 & I & $+(5)$ & $+(6)$ & $1+$ & 0.001 & 0.017 & 0.018 & $3(40,54,69)$ & I \\
\hline BR592 & 67 & G2 & $+(7)$ & $+(7)$ & $2+$ & 0.001 & 0.035 & 0.036 & $\begin{array}{c}4 \\
(48,52,55,60)\end{array}$ & I \\
\hline BR593 & 48 & G3 & $+(7)$ & $+(3)$ & $1+$ & 0.088 & 0.242 & 0.330 & $\stackrel{3}{3}(33 \mathrm{BBC}, 65,70)$ & I (24) \\
\hline BR599 & $39+44 \mathrm{CBC}$ & G3 & $+(6)$ & - $(0)$ & $3+$ & 0.187 & 0.370 & 0.557 & $2(44,60)$ & I \\
\hline BR603 & 40 & G2 & $+(7)$ & $+(7)$ & - & 0.001 & 0.032 & 0.033 & I (36) & I \\
\hline BR6I5 & 49 & GI & $+(7)$ & $+(7)$ & $1+$ & 0.000 & 0.004 & 0.004 & $2(49,50)$ & I \\
\hline BR649 & 80 & G2 & $+(7)$ & $+(6)$ & $1+$ & 0.001 & 0.404 & 0.406 & $2(3 \mathrm{I}, 44 \mathrm{BBC})$ & I \\
\hline BR650 & 47 & G3 & $+(6)$ & $-(0)$ & - & 0.004 & 0.017 & 0.021 & I (45) & I \\
\hline BR656 & 43 & G2 & $+(6)$ & $+(7)$ & $1+$ & 0.003 & 0.269 & 0.272 & $3(40,44,52)$ & I \\
\hline BR666 & 56 & G2 & $+(8)$ & $+(6)$ & $1+$ & 0.132 & 0.201 & 0.332 & $3(39,50,5 \mathrm{I})$ & $\begin{array}{c}3 \\
(49,54,61)\end{array}$ \\
\hline
\end{tabular}

$\mathrm{BC}=$ breast cancer; $\mathrm{CBC}=$ controlateral breast cancer; $\mathrm{OC}=$ ovarian cancer; $/=$ no cases; $\mathrm{M}=$ male. The ages at diagnosis of the first cousins are in boldface type; ${ }^{\circ} \mathrm{IHC}$ Allred total scores of 3 or more were reported as positive. \$HER2 IHC scores: - negative, I+ weak, 2+ moderate, 3+ strong. ^ Probability of mutation calculated by BRCAPRO software http://www.isds.duke.edu/ gp/ brcapro.html; * number of additional tumours in the family and ages of onset in parentheses. 
Table 5: Relation between breast cancer receptorial status and BRCAI mutant genotype.

\begin{tabular}{lccc}
\hline Receptorial status & BRCAI carriers & non-BRCAI cases & Total \\
\hline${ }^{\circ}$ ER- and/or PR- & 10 & 19 & 29 \\
\hline ER+/PR+ & - & 64 & 64 \\
\hline Total & 10 & 83 & 93 \\
\hline
\end{tabular}

Sensitivity 100\%; Specificity 77.1\%; Predictive Positive Value 34.4\%; Predictive Negative Value $100 \%{ }^{\circ}{ }^{I H C}$ Allred total scores of 3 or more were reported as positive.

Table 6: Relation between breast cancer receptorial status and BRCAI mutant genotype excluding HER2 3+ cases.

\begin{tabular}{lccc}
\hline Receptorial status & BRCAI carriers & non-BRCAl cases & Total \\
\hline${ }^{\circ}$ ER- and/or PR- & 10 & 12 & 22 \\
\hline ER+/PR+ & - & 60 & 60 \\
\hline Total & 10 & 72 & 82 \\
\hline
\end{tabular}

Sensitivity 100\%; Specificity 83.3\%; Predictive Positive Value 45.4\%; Predictive Negative Value $100 \%$; ${ }^{\circ}$ HC Allred total scores of 3 or more were reported as positive.

The detection rate of BRCA1 mutations was shown to increase from $10.8 \%(10 / 93)$ to $45.5 \%(10 / 22)$, thus allowing the minimization of the number of patients who should undergo BRCA1 mutation screening.

\section{Discussion}

BRCA1-associated tumours have a high probability of being ER negative (up to 90\%), PR negative (79\%) and with a low frequency of HER2 expression [4,5,19-21]. Physical co-deletion of BRCA1 and HER2 loci on chromosome $17 \mathrm{q}$, occurring as a second somatic inactivating hit, may be at least partly responsible for the low incidence of HER2 expression oramplification in BRCA1-associated carcinomas [22-24].

In the present series, four of the five triple negative tumours $(80 \%)$ harboured BRCA1 mutations. Consistently with these data, the screening carried out by Musolino et al detected 10 BRCA1 mutations: seven of the eight IHC evaluated BRCA1-related tumours had a triple negative phenotype (87\%) and all were negative for HER2 expression [25].

We report an overall prevalence of BRCA1 mutations of $10.8 \%(10 / 93)$. The FBC screening procedure based on the selection of women who were ER and/or PR negative and with weakly expressed HER2, showed a sensitivity in BRCA1 mutation carriers of $100 \%$ and a specificity of $83.3 \%$, with a positive predictive value of $45.4 \%$ and a negative predictive value of $100 \%$. Moreover, all BRCA1 mutation carriers were identified by using IHC screening, thus confirming that the Allred's method can be used to accurately predict the probability of being a carrier of the BRCA1 mutation.

The IHC test has a very high sensitivity and specificity, and may substantially improve the detection of BRCA1 pathogenetic mutations in families with hereditary breast cancer.

It has been suggested that the IHC analysis of ER may be a new powerful predictor of the BRCA1 mutation status. Estimations show that the probability of being a BRCA1mutation carrier in a woman with FBC diagnosed before the age of 35 years with a grade 3 tumour and ER negative status is $25 \%$. However, the probability falls to only $5 \%$ if the tumour is ER positive $[1,5]$.

The immunophenotypic features of breast carcinomas arising in BRCA1 mutation carriers have also been evaluated by Palacios et al, showing that only $25 \%$ of BRCA1associated tumors were ER positive and PR negative, whereas all cases were HER2 negative [24].

It should be taken into account, however, that the use of IHC screening to select cases for BRCA1 mutational analysis may have some limitations. In fact, although the majority of BRCA1-mutant tumours lack ER expression, several studies have shown that a small number retain ER positivity $[26,27]$. In addition, whereas the ER negative status is a highly sensitive predictor (ER-positive cases are 
rarely BRCA1 mutation carriers), this parameter has a limited specificity [1].

In this series of selected patients, a high prevalence of BRCA1 pathogenetic mutations was observed in FBCs with double or single negative receptorial status, showing that the evaluation of IHC characteristics can be useful not only for therapeutic purposes, but also for the identification of a subgroup of patients having a higher probability of being carriers of BRCA1 deleterious mutations. Furthermore, the evaluation of HER2 expression may also allow for a reduction of the number of patients who should undergo BRCA1 mutation screening.

\section{Conclusion}

The IHC analysis by Allred's method, a rapid and easily performed test, can be used before performing expensive mutation screening in order to select the high-risk cases who are most likely to carry a deleterious BRCA1 mutation. In patients selected by family history, the analysis of the cancer IHC phenotype allows the prediction of the BRCA1 genotype with a very high sensitivity and specificity.

About $90 \%$ of BRCA1 related cancers are ER negative and/ or PR negative with weak HER2 expression [4,5,19-21]. Although the IHC evaluation successfully allowed the limitation of the number of patients to be molecularly tested, a small proportion of cases carrying the BRCA1 mutations retained the ER expression.

Interestingly, our data confirm that the incidence of BRCA1 mutations in the ER and/or PR positive cancers is low. On these grounds, our combined selection approach could significantly reduce the number of patients who should undergo BRCA1 mutational analysis, with the possible limitation of missing one true BRCA1 mutation carrier every 100 patients selected by breast cancer familial history alone. Analysis of a larger series of cases is however required to validate the proposed selection approach.

\section{Competing interests}

The authors declare that they have no competing interests.

\section{Authors' contributions}

GM performed the genetic counselling and drafted the manuscript; AVi performed the molecular genetic studies and was involved in drafting and revising the paper; LDP performed the BRCA test; CDG, LN, SS, DLand AV collected the clinical data; $\mathrm{VC}$, TPperformed the immunohistochemical characterization; RD performed the genetic counselling and was involved in drafting and revising the paper. All authors read and approved the final manuscript.

\section{Acknowledgements}

The authors thank the patients and their families for their cooperation.

The authors also thank Anna Maria Colussi for her assistance in editing the text.

The study was supported by funds from the Italian Ministry of Health, "Ricerca Corrente" programs and by Alleanza Contro il Cancro (funding given to $\mathrm{AVi})$.

\section{References}

I. Lakhani SR, Reis-Filho JS, Fulford L, Penault-Llorca F, Vijver M van der, Parry S, Bishop T, Benitez J, Rivas C, Bignon YJ, Chang-Claude J, Hamann U, Cornelisse CJ, Devilee P, Beckmann MW, Nestle-Krämling C, Daly PA, Haites N, Varley J, Lalloo F, Evans G, Maugard C, Meijers-Heijboer H, Klijn JG, Olah E, Gusterson BA, Pilotti S, Radice P, Scherneck S, Sobol H, Jacquemier J, Wagner T, Peto J, Stratton MR, McGuffog L, Easton DF, Breast Cancer Linkage Consortium: Prediction of BRCAI status in patients with breast cancer using estrogen receptor and basal phenotype. Clin Cancer Res 2005, I I:5175-5 | 80.

2. Foulkes WD, Metcalfe K, Sun P, Hanna WM, Lynch HT, Ghadirian P, Tung N, Olopade O, Weber BL, McLennan J, Olivotto IA, Bégin LR, Narod SA: Estrogen receptor status in BRCAI- and BRCA2related breast cancer: the influence of age, grade, and histological type. Clin Cancer Res 2004, I 0:2029-2034.

3. Robson M, Gilewski T, Haas B, Levin D, Borgen P, Rajan P, Hirschaut Y, Pressman P, Rosen PP, Lesser ML, Norton L, Offit K: BRCA-associated breast cancer in young women. J Clin Oncol 1998, 1 6: 1642-1649.

4. Chappuis PO, Nethercot V, Foulkes WD: Clinico-pathological characteristics of BRCAI - and BRCA2-related breast cancer. Semin Surg Oncol 2000, 18:287-295.

5. Vaziri SA, Krumroy LM, Elson P, Budd GT, Darlington G, Myles J, Tubbs RR, Casey G: Breast tumor immunophenotype of BRCAI-mutation carriers is influenced by age at diagnosis. Clin Cancer Res 2001, 7:1937-1945.

6. Frank TS, Manley SA, Olopade OI, Cummings S, Garber JE, Bernhardt B, Antman K, Russo D, Wood ME, Mullineau L, Isaacs C, Peshkin B, Buys S, Venne V, Rowley PT, Loader S, Offit K, Robson M, Hampel H, Brener D, Winer EP, Clark S, Weber B, Strong LC, Thomas A: Sequence analysis of BRCAI and BRCA2: correlation of mutations with family history and ovarian cancer risk. J Clin Oncol 1998, 16:24I7-2425.

7. Parmigiani G, Berry D, Aguilar O: Determining carrier probabilities for breast cancer-susceptibility genes BRCAI and BRCA2. Am J Hum Genet 1998, 62: |45-I58.

8. American Society of Clinical Oncology: American Society of Clinical Oncology Policy Statement update: Genetic Testing for Cancer Susceptibility. J Clin Oncol 2003, 2 I:2397-2406.

9. Shih HA, Couch FI, Nathanson KL, Blackwood MA, Rebbeck TR, Armstrong KA, Calzone K, Stopfer J, Seal S, Stratton MR, Weber BL: $B R C A I$ and $B R C A 2$ mutation frequency in women evaluated in a breast cancer risk evaluation clinic. J Clin Oncol 2002, 20:994-999.

10. Foretova L, Machackova E, Navratilova M, Pavlu H, Hruba M, Lukesova M, Valik D: BRCAI and BRCA2 mutations in women with familial or early-onset breast/ovarian cancer in the Czech Republic. Hum Mutat 2004, 23:397-398.

II. Capalbo C, Ricevuto E, Vestri A, Ristori E, Sidoni T, Buffone O, Adamo B, Cortesi E, Marchetti P, Scambia G, Tomao S, Rinaldi C, Zani M, Ferraro S, Frati L, Screpanti I, Gulino A, Giannini G: BRCAI and BRCA2 genetic testing in Italian breast and/or ovarian cancer families: mutation spectrum and prevalence and analysis of mutation prediction models. Ann Oncol 2006, I7(Suppl 7):vii34-vii40.

12. Peto J, Collins N, Barfoot R, Seal S, Warren W, Rahman N, Easton DF, Evans C, Deacon J, Stratton MR: Prevalence of BRCAI and BRCA2 gene mutations in patients with early-onset breast cancer. J Natl Cancer Inst 1999, 91:943-949.

13. Veronesi A, de Giacomi C, Magri MD, Lombardi D, Zanetti M, Scuderi C, Dolcetti R, Viel A, Crivellari D, Bidoli E, Boiocchi M: Familial breast cancer: characteristics and outcome of BRCA I-2 positive and negative cases. BMC Cancer 2005, 5:70. 
14. Wagner TM, Hirtenlehner K, Shen P, Moeslinger R, Muhr D, Fleischmann E, Concin H, Doeller W, Haid A, Lang AH, Mayer P, Petru E, Ropp E, Langbauer G, Kubista E, Scheiner O, Underhill P, Mountain J, Stierer M, Zielinski C, Oefner P: Global sequence diversity of BRCA2: analysis of 71 breast cancer families and 95 control individuals of worldwide populations. Hum Mol Genet 1999, 8:4 13-423. Erratum in Hum Mol Genet 8:717-7|9

15. Santarosa M, Viel A, Dolcetti R, Crivellari D, Magri MD, Pizzichetta MA, Tibiletti MG, Gallo A, Tumolo S, Del Tin L, Boiocchi M: Low incidence of BRCAI mutations among Italian families with breast and ovarian cancer. Int J Cancer 1998, 78:58I-586.

16. Santarosa M, Dolcetti R, Magri MD, Crivellari D, Tibiletti MG, Gallo A, Tumolo S, Della Puppa L, Furlan D, Boiocchi M, Viel A: BRCAI and BRCA2 genes: role in hereditary breast and ovarian cancer in Italy. Int J Cancer 1999, 83:5-9.

17. The BIC (Breast Cancer Information Core) database [http:/ /www.nhgri.nih.gov/Intramural research/Lab transfer/Bic/]

18. Allred DG, Harvey JM, Belardo M, Clark GM: Prognostic and predictive factors in breast cancer by immunohistochemical analysis. Mod Pathol 1998, I I:155-168.

19. Honrado B, Benitez J, Palacios J: The molecular pathology of hereditary breast cancer: genetic testing and therapeutic implications. Mod Pathol 2005, 1 8: | 305-1320.

20. Turner NC, Reis-Filho JS: Basal-like breast cancer and the BRCAI phenotype. Oncogene 2006, 25:5846-5853.

21. Tischkowitz MD, Foulkes WD: The basal phenotype of BRCAIrelated breast cancer: past, present and future. Cell Cycle 2006, 5:963-967.

22. Jóhannsson OT, Idvall I, Anderson C, Borg A, Barkardóttir RB, Egilsson $\mathrm{V}$, Olsson $\mathrm{H}$ : Tumour biological features of BRCAIinduced breast and ovarian cancer. Eur J Cancer 1997, 33:362-37I

23. Osorio A, de la Hoya M, Rodriguez-Lopez R, Martínez-Ramírez $A$ Cazorla A, Granizo J], Esteller M, Rivas C, Caldés T, Benítez J: Loss of heterozygosity analysis at the BRCA loci in tumor samples from patients with familial breast cancer. Int J Cancer 2002, 99:305-309.

24. Palacios J, Honrado E, Osorio A, Cazorla A, Sarrió D, Barroso A, Rodríguez S, Cigudosa JC, Diez O, Alonso C, Lerma E, Sánchez L, Rivas C, Benítez J: Immunohistochemical characteristics defined by tissue Microarray of Hereditary Breast Cancer not attributable to BRCAI or BRCA2 mutations: differences from breast carcinomas arising in BRCAI and BRCA2 mutation carriers. Clin Cancer Res 2003, 9:3606-3614.

25. Musolino A, Bella AM, Bortesi B, Michiara M, Naldi N, Zanelli P, Capelletti M, Pezzuolo D, Camisa R, Savi M, Neri TM, Ardizzoni A: BRCA mutations, molecular markers, and clinical variables in early-onset beast cancer: a population-based study. The Breast 2007, 16:280-292.

26. Karp SE, Tonin PN, Begin LR, Martinez J], Zhang JC, Pollak MN, Foulkes WD: Influence of BRCAI mutations on nuclear grade and estrogen receptor status of breast carcinoma in Ashkenazi Jewish women. Cancer 1997, 80:435-44I.

27. Verhoog LC, Brekelmans CT, Seynaeve C, Bosch LM van den, Dahmen G, van Geel AN, Tilanus-Linthorst MM, Bartels CC, Wagner A Ouweland A van den, Devilee P, Meijers-Heijboer EJ, Klijn JG: Survival and tumour characteristics of breast-cancer patients with germline mutations of BRCAI. Lancet 1998, 35 I:316-321.

\section{Pre-publication history}

The pre-publication history for this paper can be accessed here:

http://www.biomedcentral.com/1471-2407/9/360/pre pub
Publish with Biomed Central and every scientist can read your work free of charge

"BioMed Central will be the most significant development for disseminating the results of biomedical research in our lifetime. "

Sir Paul Nurse, Cancer Research UK

Your research papers will be:

- available free of charge to the entire biomedical community

- peer reviewed and published immediately upon acceptance

- cited in PubMed and archived on PubMed Central

- yours - you keep the copyright
BioMedcentral 